\title{
Time-dependent changes in the expression of lymphocyte and monocyte cell adhesion molecules after meals of different composition
}

\author{
Esther Torrecilla ${ }^{1}$, Miguel González-Muñoz ${ }^{2}$, Carlos Lahoz ${ }^{1}$ and Jose $\operatorname{Mostaza}^{1} *$ \\ ${ }^{1}$ Atherosclerosis Unit, Hospital Carlos III, Madrid, Spain \\ ${ }^{2}$ Immunology Department, Hospital Carlos III, Madrid, Spain
}

(Received 15 October 2009 - Revised 6 June 2010 - Accepted 8 June 2010 - First published online 9 August 2010)

\begin{abstract}
The objective of the present study was to compare the acute effect of meals of different composition on the expression of adhesion molecules that play a key role in leucocyte trafficking. A total of twenty apparently healthy subjects randomly consumed three isoenergetic meals 1 week apart: enriched in carbohydrates (CHO), enriched in monounsaturated fat and enriched in saturated fat. Blood samples were obtained before the meals and at 2, 4, 6, 8 and $10 \mathrm{~h}$ after meal ingestion. Samples were analysed for LDL resistance to Cu-mediated oxidation and for the surface expression on peripheral blood mononuclear cells (PBMC) of CD62L, CD162, CD11a, CD11b, CD49d and CD54 by flow cytometry. The present results showed that there were no changes in LDL susceptibility to oxidation within and among the meals. After the CHO-enriched meal, there was a time-dependent increased expression of CD162, CD49d, CD11a and CD54 on PBMC that returned to basal values after 8-10h. These changes were significantly greater than the ones observed after the consumption of the monounsaturated fat- and the saturated fat-enriched meals and were more evident in lymphocytes than in monocytes. In conclusion, acute ingestion of a CHO-enriched meal induces higher increases of lymphocyte activation markers than fat-enriched meals. These results suggest that long-term consumption of CHO-enriched diets may be associated with a sustained pro-inflammatory state.
\end{abstract}

Monocytes: Lymphocytes: Adhesion molecules: Diet: Carbohydrates: Monounsaturated fat: Saturated fat: Postprandial state

Both postprandial glycaemia ${ }^{(1,2)}$ and postprandial lipaemia ${ }^{(3)}$ are markers of increased vascular risk. After meal ingestion, the components of some foods could negatively influence a number of metabolic or cellular pathways which, repetitively and in the long term, could promote the development of atherosclerosis. This relationship has generally been ascribed to the effect of diet composition on metabolic factors epidemiologically linked to CVD. However, the direct effect that the diet could exert on cells involved in the atherogenic process has received less attention.

Atherosclerosis is a chronic inflammatory disease in which adhesion molecules of peripheral blood mononuclear cells (PBMC) play a key role in different stages of its development $^{(4)}$. L-selectin (CD62L) mediates leucocyte adhesion and rolling on the endothelial surface ${ }^{(5)}$, molecules of the $\beta$-integrin family, CD11a, CD49d and CD11b, are responsible for tight leucocyte attachments to endothelial receptors and, in a third stage, intercellular adhesion molecule-1 (ICAM-1) (CD54) interacts with its ligands of the extracellular matrix promoting PBMC transmigration into the subendothelial space $^{(6)}$.

The aim of the present study was to evaluate the acute effects of meals of different consumption on PBMC activation assessed by changes in the expression of key adhesion molecules implicated in leucocyte trafficking into the vessel wall.

\begin{abstract}
Methods and volunteers
The present study was a randomised, cross-over study, performed in twenty normolipaemic subjects (seven men; mean age 32 years; age range 26-40 years; BMI 24.3 (SD 2.5) $\mathrm{kg} / \mathrm{m}^{2}$ ). Participants were healthy volunteers recruited from the staff of the Hospital Carlos III. They were summoned at 07.30 hours after a $12 \mathrm{~h}$ fast, for three consecutive Mondays. Upon arrival, an intravenous cannula was placed for venous blood sampling. After the first blood extraction (time 0), and according to a list of randomisation, participants ingested one of three mixed, isoenergetic meals: rich in carbohydrates (CHO), rich in monounsaturated fat; rich in saturated fat. The meal rich in $\mathrm{CHO}$ contained $50 \mathrm{~g}$ white wheat bread, $400 \mathrm{ml}$ skimmed milk, $40 \mathrm{~g}$ white sugar, $40 \mathrm{~g}$ strawberry jam and $60 \mathrm{~g}$ chocolate powder, had an energy content of $3231 \mathrm{~kJ}$, a glycaemic index of 56, a glycaemic load of $84.5,84 \%$ energy as CHO, $11 \%$ energy as protein, $2.2 \%$ energy as saturated fat, $1.2 \%$ energy as monounsaturated fat and $0.3 \%$ energy as polyunsaturated fat. The fatty acid composition of the high-CHO meal was: $14: 0,0 \mathrm{~g} ; 16: 0,1 \mathrm{~g} ; 18: 0,1.2 \mathrm{~g}$;
\end{abstract}

Abbreviations: CHO, carbohydrate; FITC, fluorescein isothiocyanate; PBMC, peripheral blood mononuclear cells; PE, phycoerythrin. 
$16: 1,0 \mathrm{~g} ; 18: 1,1.2 \mathrm{~g} ; 18: 2,0 \cdot 3 \mathrm{~g} ; 18: 3,0 \mathrm{~g}$; EPA and DHA, $0 \mathrm{~g}$. The meal rich in monounsaturated fat contained $50 \mathrm{~g}$ white wheat bread, $36 \mathrm{~g}$ olive oil, $400 \mathrm{ml}$ skimmed milk and $30 \mathrm{~g}$ hazelnuts, had an energy content of $3327 \mathrm{~kJ}, 25 \%$ energy as $\mathrm{CHO}, 11 \%$ energy as protein, $6.7 \%$ energy as saturated fat, $40.6 \%$ energy as monounsaturated fat and $6.2 \%$ energy as polyunsaturated fat. The fatty acid composition of the high-monounsaturated fat meal was: $14: 0,0 \mathrm{~g}$; $16: 0,5.1 \mathrm{~g} ; 18: 0,1.4 \mathrm{~g} ; 16: 1,0.4 \mathrm{~g} ; 18: 1,40 \mathrm{~g} ; 18: 2$, $5.9 \mathrm{~g} ; 18: 3,0.3 \mathrm{~g}$; EPA and DHA, $0 \mathrm{~g}$. The meal rich in saturated fat contained $50 \mathrm{~g}$ white wheat bread, $40 \mathrm{~g}$ butter, $400 \mathrm{ml}$ whole milk and $30 \mathrm{~g}$ cheese, had an energy content of $3273 \mathrm{~kJ}$, $24 \%$ energy as $\mathrm{CHO}, 12 \%$ energy as protein, $32.8 \%$ energy as saturated fat, $16.2 \%$ energy as monounsaturated fat and $1.6 \%$ energy as polyunsaturated fat. The fatty acid composition of the high-saturated fat meal was: $14: 0,6 \cdot 1 \mathrm{~g} ; 16: 0$, $16 \cdot 1 \mathrm{~g} ; 18: 0,6.5 \mathrm{~g} ; 16: 1,1.7 \mathrm{~g} ; 18: 1,14 \mathrm{~g} ; 18: 2,1 \mathrm{~g}$; $18: 3,0.6 \mathrm{~g}$; EPA and DHA, $0 \mathrm{~g}$. After meal ingestion the volunteers were only allowed to consume mineral water. Blood samples were obtained at 2, 4, 6, 8 and $10 \mathrm{~h}$ after the first blood extraction.

Cholesterol and TAG concentrations were determined from plasma using enzymic colorimetric methods. HDLcholesterol was measured after precipitation of apoB lipoproteins with phosphotungstate- $\mathrm{MgCl}_{2}$. LDL were isolated after sequential ultracentrifugation, oxidised with $\mathrm{Cu}_{2} \mathrm{SO}_{4}$ and conjugated dienes were measured as previously described $^{(7)}$.

Leucocyte activation markers were measured from heparinised blood within $1 \mathrm{~h}$ of venepuncture. A quantity of $50 \mu \mathrm{l}$ of each sample was incubated with the following monoclonal antibody mixtures: CD11a (clone G-25.2, IgG2a)-fluorescein isothiocyanate (FITC); CD49d (clone L25, IgG2b)-phycoerythrin (PE); CD62L (clone SK11, IgG2a)-FITC; CD54 (clone LB-2, IgG2b)-PE; CD11b (clone ICRF44, IgG1)-FITC; CD162 (clone KPL-1, IgG1)-PE. CD14 (clone MФP9, IgG2b)-peridinin chlorophyll protein (PerCP) was added to all assays. Samples were also incubated with isotypic control monoclonal antibodies to detect non-specific staining. Monoclonal antibodies were provided by $\mathrm{BD}$ Biosciences (Erembodegem, Belgium) except CD11b-FITC, provided by AbD Serotec Inc. (Oxford, Oxon, UK). A quantity of $1 \mathrm{ml}$ FACS Lysing solution (BD Biosciences) was added for $15 \mathrm{~min}$ at room temperature. After centrifugation and washing with PBS, cells were re-suspended with $300 \mu$ of $1 \%$ paraformaldehyde and acquired in a FACScan flow cytometer (BD Biosciences). A quantity of $20 \mu \mathrm{l}$ of each monoclonal antibody, except for CD11b (5 $\mu 1)$, was added to the samples.

Lymphocytes were gated according to their light-scattering characteristics and monocytes according to their high CD14 expression. At least $1000 \mathrm{CD}^{++} 4^{+}$cells and 5000 lymphocytes were analysed with CellQuest Pro software (BD Biosciences, San Jose, CA, USA). Fluorescence intensity of each cellular population was expressed as the mean fluorescence intensity (MFI) in arbitrary units. All samples were processed blinded to the type of diet consumed.

Results are presented as mean values with their standard errors. For each adhesion molecule, repeated-measures ANOVA was used for testing the effect of time, diet and time-diet interactions, after adjustment for sex, age, BMI

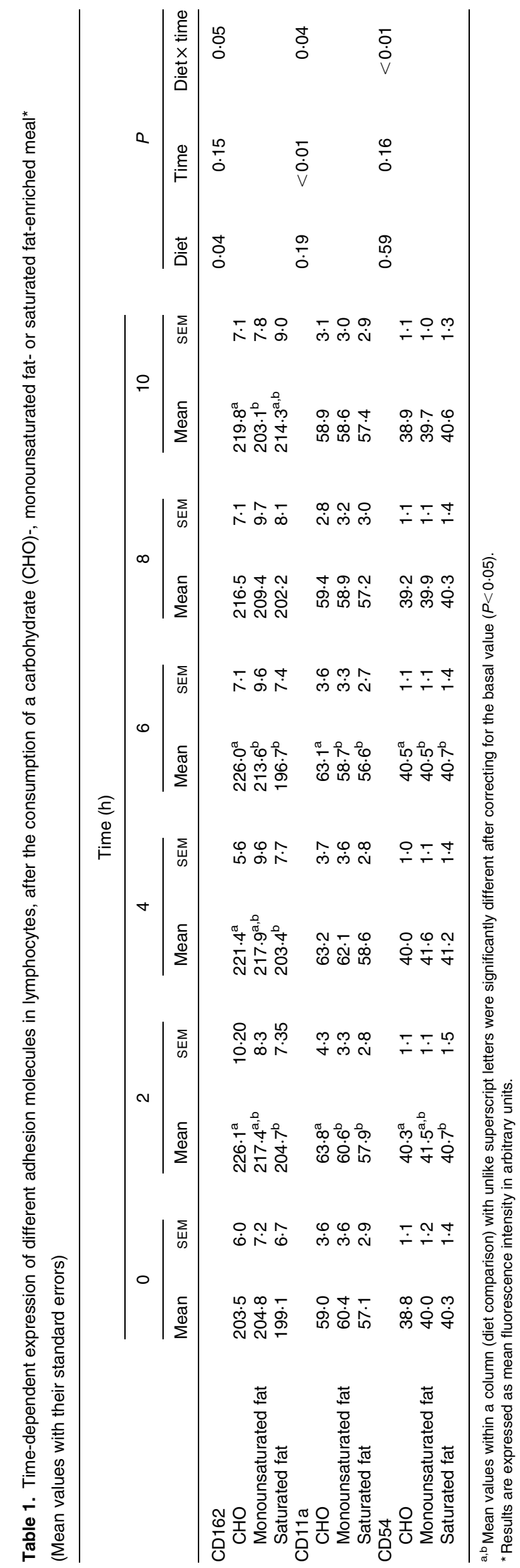


and their MFI baseline values. When statistical significance was found, Bonferroni's test was used for post hoc comparisons. The Greenhouse-Geisser statistic was used when the sphericity assumption was not satisfied. SPSS (version 15.0; SPSS, Inc., Chicago, IL, USA) was used for statistical analysis.

The present study was conducted according to the guidelines laid down in the Declaration of Helsinki and all procedures involving human subjects were approved by the Clinical Trials Ethics Committee of the Hospital Carlos III (Madrid, Spain). Written informed consent was obtained from all subjects.

\section{Results}

All diets raised plasma TAG. The increase was significantly higher with both of the fat-enriched meals. There were neither significant time changes nor differences among the diets on time to LDL oxidation ex vivo.

In lymphocytes, the expression of CD162, CD11a and CD54 increased after the ingestion of the CHO-enriched meal in comparison with both of the fat-enriched meals (Table 1). The CD11a increase was due to a raise in the percentage of lymphocytes expressing high levels of CD11a (CD11a-bright lymphocytes), returning to baseline values at $\mathrm{t}=8 \mathrm{~h}$ (Fig. 1). There were no changes in the expression of CD62L, CD11b or CD49d with any of the diets. Lymphocytes did not change their size at the different time points after meal consumption.

In monocytes, we only observed a differential expression of CD49d after the ingestion of the CHO diet compared with the enriched-fat diets (time $\times$ diet interaction, $P=0 \cdot 035$ ) (results not shown).

\section{Discussion}

The present report is the first to compare the effect of mixed meals of different composition on the expression of key adhesion molecules implicated in the atherogenic process. The present results demonstrate that an acute consumption of a CHO-enriched meal increases the expression of CD162, CD49d, CD11a and CD54 on PBMC. Globally, these timedependent changes were of greater magnitude than the ones observed after fat-enriched meals, either monounsaturated or saturated, and were more evident in lymphocytes than in monocytes. A similar, although more marked, activation
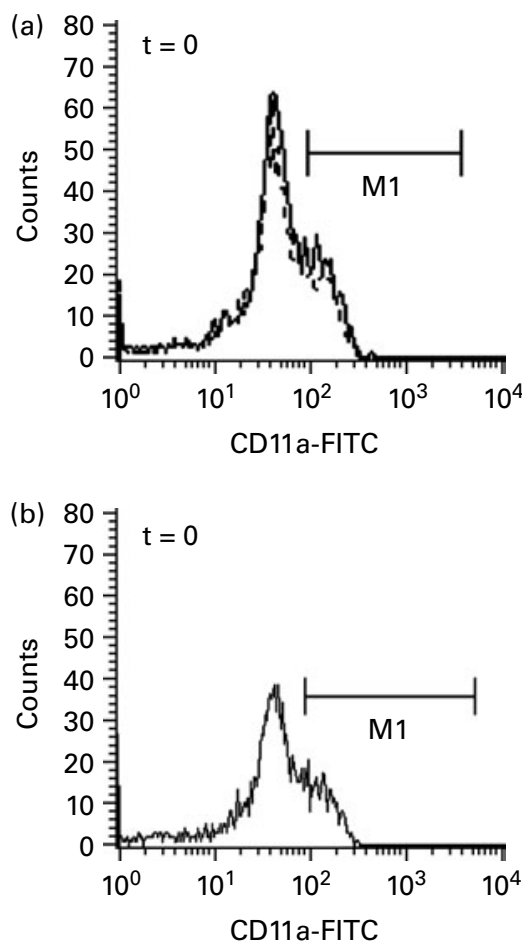
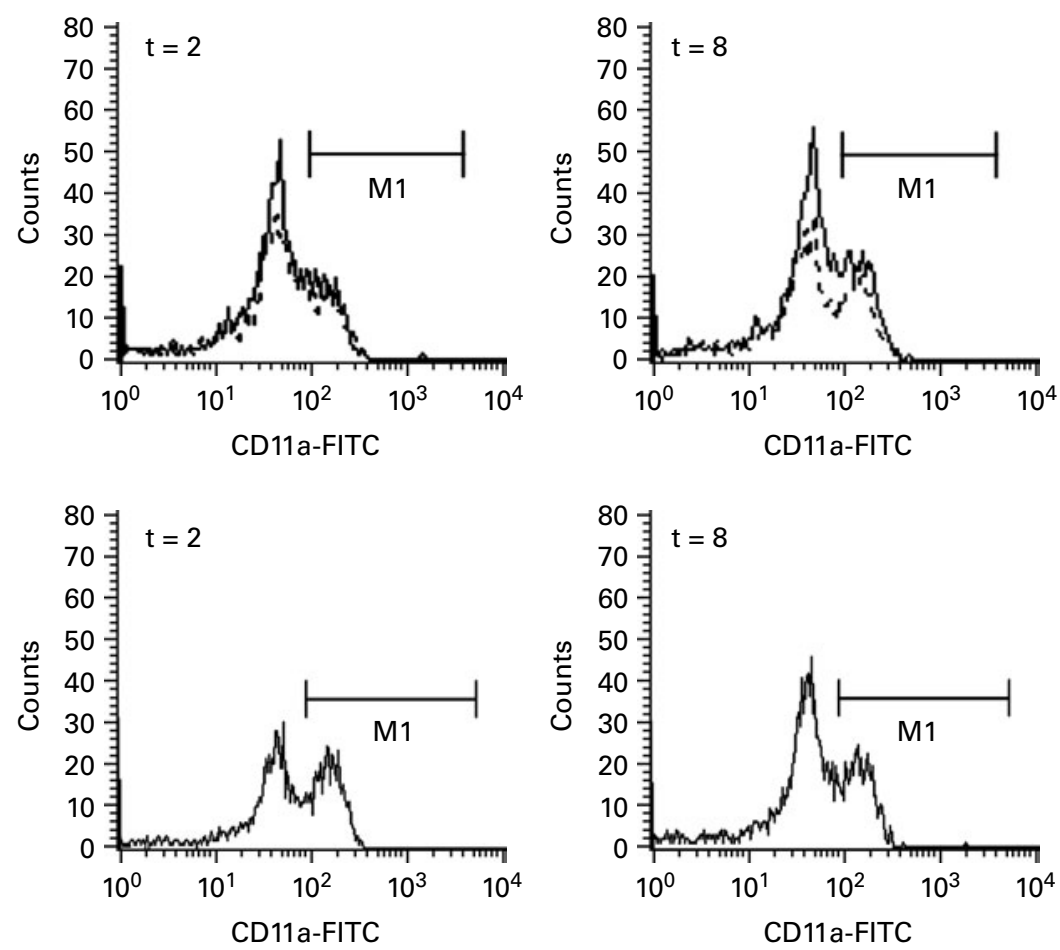

(c)

\begin{tabular}{|l|c|c|c|}
\hline \multirow{2}{*}{ Meal } & \multicolumn{3}{|c|}{ Bright CD11a lymphocytes (percentage/MFI) } \\
\cline { 2 - 4 } & $\mathrm{t}=0$ & $\mathrm{t}=2$ & $\mathrm{t}=8$ \\
\hline CHO & $24 / 136$ & $44 / 152$ & $32 / 138$ \\
\hline MUFA & $25 / 131$ & $27 / 132$ & $30 / 139$ \\
\hline SFA & $25 / 131$ & $25 / 133$ & $28 / 136$ \\
\hline
\end{tabular}

Fig. 1. Changes in the percentage of lymphocytes expressing high levels of CD11a (CD11a-bright lymphocytes; M1) after consumption of meals enriched in carbohydrate (CHO), saturated fat (SFA) and monounsaturated fat (MFA). (a) Histograms represent changes following monounsaturated fat ( - ) and saturated fat (---) diets. (b) Histograms show changes after the $\mathrm{CHO}$ diet. Three time points ( $\mathrm{t}=0,2$ and $8 \mathrm{~h}$ ) are depicted. FITC, fluorescein isothiocyanate. (c) Percentage and mean fluorescence intensity (MFI) of CD11a-bright lymphocytes. 
pattern of expression of the adhesion molecules has been described in subjects with inflammatory diseases ${ }^{(8)}$, coronary artery disease $\mathrm{e}^{(9,10)}$ and in individuals with cardiovascular risk factors ${ }^{(11,12)}$. Moreover, this phenotypic pattern has been associated with an increased adhesiveness of PMBC to the endothelium ${ }^{(13)}$. Although the observed changes are small in magnitude, a repetition of this pattern whenever a meal is consumed could, in the long term, have adverse consequences. In fact, it has been shown that chronic consumption of high$\mathrm{CHO}$ diets with a high dietary glycaemic load and glycaemic index are associated with an increased cardiovascular risk $^{(14,15)}$.

Previous studies have demonstrated that either chronic hyperglycaemia or ex vivo cellular exposure to glucose induces changes in the expression of adhesion molecules ${ }^{(16)}$ and stimulates leucocyte adhesion to the endothelium ${ }^{(17)}$ by a NF-кB-mediated mechanism ${ }^{(18)}$. Also, acute CHO consumption induces monocyte activation, as assessed by an increased expression of TNF $\alpha$, IL- $\beta^{(19)}$, CD11a, CD11b and CD54 $4^{(20)}$, and endothelial dysfunction ${ }^{(21,22)}$.

The effect of both fat-enriched meals on adhesion molecule expression was small. Most studies evaluating the effect of high-fat diets on PBMC have administered a fat load instead of a mixed fat-enriched meal. It has previously been demonstrated that the addition of either proteins ${ }^{(23)}$ or glucose $^{(24,25)}$ to a high-fat meal can modify its effect.

We conclude that the acute consumption of a mixed meal enriched in $\mathrm{CHO}$ is associated with up-regulation of adhesion molecules on circulating lymphocytes. This effect was higher than the one observed after monounsaturated and saturated fat consumption. These potentially harmful findings could contribute to the increased cardiovascular risk that has been attributed to high-CHO diets.

\section{Acknowledgements}

The present study has been funded by a research grant from Fondo de Investigaciones Sanitarias, Instituto de Salud Carlos III (no. 03/1211) and from Fundación para el fomento y Desarrollo de la Investigación Clínica.

J. M. conceived of and designed the study, interpreted the data and drafted the article; M. G.-M. and C. L. conceived of and designed the study, interpreted the data and revised the article; E. T. was in charge of all the laboratory work, interpreted the data and revised the article.

There are no conflicts of interest.

\section{References}

1. Howlett J \& Ashwell M (2008) Glycemic response and health: summary of a workshop. Am J Clin Nutr 87, 212S-216S.

2. Cavalot F, Petrelli A, Traversa M, et al. (2006) Postprandial blood glucose is a stronger predictor of cardiovascular events than fasting blood glucose in type 2 diabetes mellitus, particularly in women: lessons from the San Luigi Gonzaga Diabetes Study. J Clin Endocrinol Metab 91, $813-819$.

3. Lopez-Miranda J, Perez-Martinez P, Marin C, et al. (2006) Postprandial lipoprotein metabolism, genes and risk of cardiovascular disease. Curr Opin Lipidol 17, 132-138.
4. Ross R (1999) Atherosclerosis - an inflammatory disease. $N$ Engl J Med 340, 115-126.

5. Rainer TH (2002) L-selectin in health and disease. Resuscitation 52, $127-141$.

6. Carlos TM \& Harlan JM (1994) Leukocyte-endothelial adhesion molecules. Blood 84, 2068-2101.

7. Esterbauer H, Striegl G, Puhl H, et al. (1989) Continuous monitoring of in vitro oxidation of human low density lipoprotein. Free Radic Res Commun 6, 67-75.

8. Bhatnagar A, Wig JD \& Majumdar S (2001) Expression of activation, adhesion molecules and intracellular cytokines in acute pancreatitis. Immunol Lett 77, 133-141.

9. Kawamura A, Miura S, Murayama T, et al. (2004) Increased expression of monocyte CD11a and intracellular adhesion molecule-1 in patients with initial atherosclerotic coronary stenosis. Circ J 68, 6-10.

10. Meisel SR, Shapiro H, Radnay J, et al. (1998) Increased expression of neutrophil and monocyte adhesion molecules LFA-1 and Mac-1 and their ligand ICAM-1 and VLA-4 throughout the acute phase of myocardial infarction: possible implications for leukocyte aggregation and microvascular plugging. J Am Coll Cardiol 31, 120-125.

11. Weber C, Erl W, Weber KS, et al. (1997) HMG-CoA reductase inhibitors decrease CD11b expression and CD11b-dependent adhesion of monocytes to endothelium and reduce increased adhesiveness of monocytes isolated from patients with hypercholesterolemia. J Am Coll Cardiol 30, 1212-1217.

12. van Oostrom AJ, Van Wijk JP, Sijmonsma TP, et al. (2004) Increased expression of activation markers on monocytes and neutrophils in type 2 diabetes. Neth J Med 62, 320-325.

13. van Gils JM, Zwaginga JJ \& Hordijk PL (2009) Molecular and functional interactions among monocytes, platelets, and endothelial cells and their relevance for cardiovascular diseases. J Leukoc Biol 85, 195-204.

14. Beulens JW, de Bruijne LM, Stolk RP, et al. (2007) High dietary glycemic load and glycemic index increase risk of cardiovascular disease among middle-aged women: a population-based follow-up study. J Am Coll Cardiol 50, $14-21$.

15. Liu S, Willett WC, Stampfer MJ, et al. (2000) A prospective study of dietary glycemic load, carbohydrate intake, and risk of coronary heart disease in US women. Am J Clin Nutr 71, $1455-1461$.

16. Kado S, Wakatsuki T, Yamamoto M, et al. (2001) Expression of intercellular adhesion molecule-1 induced by high glucose concentrations in human aortic endothelial cells. Life Sci $\mathbf{6 8}$, $727-737$.

17. Kim JA, Berliner JA, Natarajan RD, et al. (1994) Evidence that glucose increases monocyte binding to human aortic endothelial cells. Diabetes 43, 1103-1107.

18. Morigi M, Angioletti S, Imberti B, et al. (1998) Leukocyteendothelial interaction is augmented by high glucose concentrations and hyperglycemia in a NF-кB-dependent fashion. J Clin Invest 101, 1905-1915.

19. Motton DD, Keim NL, Tenorio FA, et al. (2007) Postprandial monocyte activation in response to meals with high and low glycemic loads in overweight women. Am J Clin Nutr $\mathbf{8 5}$, 60-65.

20. Sampson MJ, Davies IR, Brown JC, et al. (2003) Monocyte and neutrophil adhesion molecule expression during acute hyperglycemia and after antioxidant treatment in type 2 diabetes and control patients. Arterioscler Thromb Vasc Biol 22, 1187-1193.

21. Kawano H, Motoyama T, Hirashima O, et al. (1999) Hyperglycemia rapidly suppresses flow-mediated endothelium-dependent vasodilation of brachial artery. J Am Coll Cardiol 34, $146-154$. 
22. Title LM, Cummings PM, Giddens K, et al. (2000) Oral glucose loading acutely attenuates endothelium-dependent vasodilation in healthy adults without diabetes: an effect prevented by vitamins C and E. J Am Coll Cardiol 36, 2185-2191.

23. Westphal S, Taneva E, Kastner S, et al. (2006) Endothelial dysfunction induced by postprandial lipemia is neutralized by addition of proteins to the fatty meal. Atherosclerosis 185, $313-319$.
24. van Oostrom AJ, van Dijk H, Verseyden C, et al. (2004) Addition of glucose to an oral fat load reduces postprandial free fatty acids and prevents the postprandial increase in complement component. Am J Clin Nutr 79, 510-515.

25. Knuth ND, Remias DB \& Horowitz JF (2008) Adding carbohydrate to a high-fat meal blunts postprandial lipemia in women and reduces meal-derived fatty acids in systemic circulation. Appl Physiol Nutr Metab 33, 315-325. 